\title{
A Study of Routing Path Decision Method Using Mobile Robot Based on Distance Between Sensor Nodes
}

\author{
Yuta Koike \\ Department of Information and \\ Communication \\ Engineering, Tokyo Denki \\ University \\ Tokyo, Japan
}

\author{
Kei Sawai \\ Department of Information and \\ Communication \\ Engineering, Tokyo Denki \\ University \\ Tokyo, Japan
}

\author{
Tsuyoshi Suzuki \\ Department of Information and \\ Communication \\ Engineering, Tokyo Denki \\ University \\ Tokyo, Japan
}

\begin{abstract}
We propose Robot Wireless Sensor Networks (RWSNs) management method for maintaining wireless communication connectivity for a mobile robot teleoperation with considering a distance between sensor nodes. Recent studies for reducing disaster damage focus on a disaster area information gathering in underground spaces. Since information gathering activities in such post disaster underground spaces present a high risk of personal injury by secondary disasters, a lot of rescue workers were injured or killed in the past. On basis of this background, gathering information by utilizing the mobile robot is discussed in wide area. However, maintaining wireless communication infrastructures for teleoperation of a mobile rescue robot in the post-disaster underground space by various reasons. Therefore we have been discussing the wireless communication infrastructures construction method for teleoperation of the rescue robot by utilizing the RWSN. In this paper, we evaluated the proposed method for changing routing path by utilizing the RWSN in field operation test in order to confirm the availability of performance of communication connectivity and the throughputs between End-to-End communications via constructed network.
\end{abstract}

Keywords-Wireless Sensor Networks; Moblie Robot TeleOperation; Maintaining Throughput; Routing Path

\section{INTRODUCTION}

Gathering information in disaster areas is very important for assessing the situation, avoiding secondary disasters and managing disaster reduction [1]-[7]. However, if a disaster occurs in a congested city, the rescue team cannot gather information because of the complicated urban structure. In general, gathering information from a bird's eye view with an unmanned air vehicle (UAV) is a useful method in a disaster area. However, in an urban area with many underground spaces where information gathering by using a UAV is difficult, checking on the extent of the damage, which is important for avoiding secondary disasters, is difficult [8]. Also, rescue teams cannot organize a rescue plan for underground spaces. In this situation, the rescue team has to gather damage information by entering into the underground spaces directly and share them. However, when the communication infrastructure is broken, rescue teams cannot cooperate because of disconnect between above-ground and underground spaces.
Therefore, rescue workers face secondary disaster risks increasing by a sudden situation changes. For example, in the underground disasters in Korea in 2003, many casualties occurred among rescue workers because of smoke damage. The rescue workers could not expect the smoke damage because they could not gather enough disaster area information beforehand. This is a typical case of underground secondary disaster that occurred due to the rescue team having entered into underground space without adequate information. In the future, disaster area information gathering in a closed area such as an underground spaces is important to contribute the reducing secondary disaster risks and formulating an appropriate rescue plan. On the basis of above background, recent researches have focused on disaster area information gathering method using wireless sensor networks (WSNs) in closed areas. Thus we have proposed robot wireless sensor networks (RWSNs) that include the WSN and a mobile rescue robot.

The WSN consists of spatially distributed sensor nodes (SNs) to cooperatively monitor physical or environmental conditions, such as temperature, sound, vibration, pressure, motion and so on. Then, the WSNs can provide a wireless communication infrastructure in place without established communication infrastructures. Therefore, the WSN is discussed as one of methods to construct the communication infrastructure and gather information in disaster area. However, existing construction method of the WSN is difficult to deploy $\mathrm{SN}$ in underground space such as post-disaster environment where rescue worker cannot enter.

The RWSN enables construction of wireless communication infrastructure by using the WSN and the mobile rescue robot in post-disaster underground space (Fig. 1). In our WSN deployment method for constructing the RWSN, we adopt a method that the mobile rescue robot deploys SNs which are wirelessly connected by defined routing path in advance. The mobile rescue robot moves into the underground space, and deploys SNs onto the own movement path. Deployed SN is connected to adjacent SNs wirelessly one by one, and then the WSN is expanded in underground space. An operator remotely controls the mobile rescue robot via constructed WSN communication infrastructure. In the 
network topology of this WSN, each SN is linearly connected to prevent the error of routing control. Generally, the WSN is able to decide the routing path of data transfer automatically by utilizing the RSSI between SNs, the throughput of End-to-End communication or the rate of packet loss. The routing path of the WSN is reconstructed by changes of these communication qualities. However, the routing path is repeatedly reconstructed in the situation that the communication connection between SNs is disconnected frequently. This situation is a problem for the system of the mobile robots teleoperation. An abeyance of the wireless communication connection degrades an operability of the mobile robot teleoperation and the performance of the disaster area information gathering. Then the communication qualities are often changed in disaster area by the disaster damages, the routing path is repeatedly reconstructed in the WSN. Therefore, in order to prevent the decline of the mobile robot activity, we adopt the network topology that routing path of the WSN is linearly connected. For such system, this paper describes a strategy of a routing path decision method using a mobile rescue robot for maintenance of the communication quality between SNs.

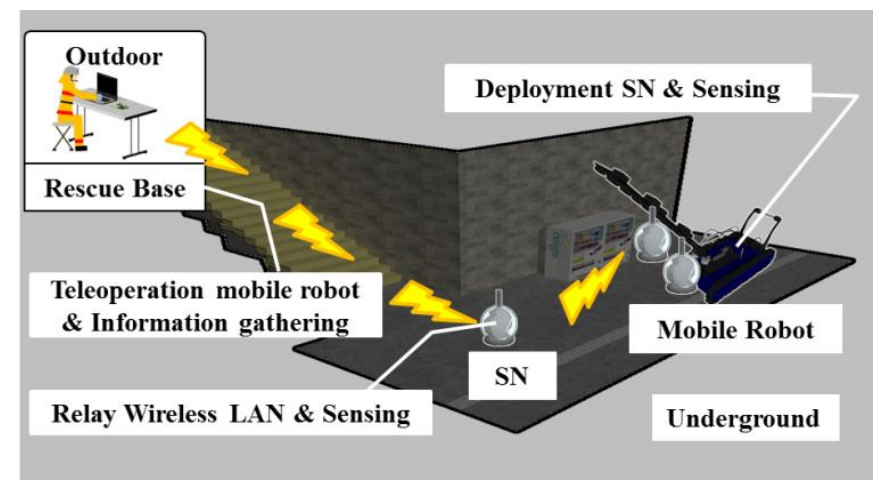

Fig. 1. Information-gathering system utilizing RWSN

\section{RELATED WORKS}

WSN construction methods have been discussed in the many research fields. In these researches, deployment methods have been proposed based on considering evaluation scales of factors such as packet routing, communication connectivity, energy efficiency, and coverage area[9]-[14].

Kumer et al. proposed a scheme for homogeneous distribution of randomly deployed mobile sensor networks to achieve maximum coverage while maintaining connectivity [15]. In this scheme, to achieve the maximum coverage and better connectivity, mobile nodes deploy themselves at the point which is divided other node's communication range into six. Wang et al. proposed a shortest moving path algorithm for the mobile robot to deploy a linear WSN [16]. This algorithm considers that the mobile robot has to return starting point to reload sensors and impact of different deployment strategies. Rizzo et al. proposed the deployment method of a robot team in fading environments [17]. In this method, to maintain constant connectivity and high signal quality in the communication network formed by the robots and the base station, the robot deployment is driven by RSSI measurements.

However, these methods don't consider an End-to-End network connectivity or a communication quality such as throughput. Many researches are premised on communication link being maintained automatically. Moreover, it is possible that the routing path reconstruction is difficult to maintain the communication quality, when the communication connection is disconnected by multi path fading in underground spaces. Therefore, considering routing path and communication quality in the WSN are important to maintain the End-to-End network connectivity.

\section{Managemaent Method Of Maintaining CommunicATION CONNECTIVITY UTILIZING MobILE Robot}

\section{A. Prior Conditions}

In our proposed system, the SNs are deployed by the mobile rescue robot to construct the WSN. An assumed environment where the WSN is constructed has entrance stairs and the first basement floor.

The entrance stairs in under-ground is required to set up at intervals 30 [m], and passage way is built in line in Japanese building standard low. Therefore, our proposed system gathers this area's information. Then we discussed the WSN construction method by utilizing the mobile robot in this area. In the wireless communication of the RWSN, IEEE 802.11 series are adopted for wireless communication between SNs including the mobile rescue robot, which has been used as proven communication protocol in many studies of mobile robots and WSNs [18]-[22]. Then in our proposed method, we treat a mobile robot as a $\mathrm{SN}$ in the WSN. Heterogeneous networks includingsome SNs and various mobile robots are difficult to manage the system control. Especially, maintaining the stability of the system control is not easy under disaster situation in underground spaces. In this environment, to simplify the network structure is necessary to construct the stable communication connection. Therefore we simplified the network structure by treating a mobile robot as a SN. From here onwards, the IEEE802.11 series is also adopted for communication system of the mobile robot in the RWSN.

In our SN deployment method for constructing the WSN, the mobile rescue robot delivers the previously wireless connected SNs. The mobile robot deploys them onto the own movement path to construct the WSN. The operator can control the mobile rescue robot via constructed WSN communication infrastructure. In the network topology of this RWSN, each SN is linearly connected for prevention of the routing control error. As mentioned before, we adopt the network topology that the SNs are linearly connected, and the routing path is defined beforehand to prevent the decline of the mobile rescue robot activity when constructing the WSN.

\section{B. Requested Specification}

IEEE 802.11 series are necessary to keep the throughput that is more than $1.0[\mathrm{Mbps}]$ between the operator and the mobile robot (End-to-End communication) [23]. The throughput is defined as the number of packet transferred per unit time in a communication network. In the WSN construction by utilizing the mobile robot, the throughput between End-to-End communications has to be maintained for comfortable mobile robot teleoperation. The construction length of the WSN is required 50 [m] by concerning the distance of first basement floor 30 [m] and entrance stairs 20 
[m]. The communication connectivity by IEEE802.11 series, however, is characterized by decreasing in turn area covered with concrete material such as the underground space. Thus, for WSN construction, our proposed system considers this communication characteristic to avoid a network disconnection risks.

The communication system among the RWSN uses IEEE802.11b standard that is tolerant to communication disruption from obstacles. The theoretical values of throughput by using IEEE802.11b are 11.0 [Mbps], and then the actual measurement values are lowered around $7.0[\mathrm{Mbps}]$ by the efficiency of the various factors in the real environment. This wireless LAN protocol can connect to SNs within 100 [m] in 1hop communication on the straight line. The throughput is required $1.0[\mathrm{Mbps}]$ or more for the mobile robot teleoperation with keeping high communication connectivity.

In the ad-hoc network constructed on IEEE802.11b communication link, the maximum number of SNs to maintain 1.0 [Mbps] or more is five, and then throughput between each SN should be kept 6.0 [Mbps] or more. In the ad-hoc networks constructing the WSN, the delay of data transfer increases with increasing the number of hops between the source and the destination SNs. In order to expand a communication distance with maintaining the throughput in the WSN construction, the necessary number of SNs is decided beforehand, and then the routing path of these $\mathrm{SNs}$ is connected linearly. This method provides the high connectivity in an area covered with concrete material such as the underground space by deploying SNs as communication relay nodes to construct the WSN. In this system, the RWSN is constructed by using a source SN for the operator, three SNs for communication relay deployed by the mobile robot and the mobile robot regarded as one SN.

\section{SN Deployment method for maintaining communication quality}

In our proposed method, the mobile robot simultaneously measures communication quality while moving in the environment, and decides the deployment position of a $\mathrm{SN}$. Therefore, the SN position can be determined flexibly against a change of radio wave condition, the mobile robot can cover with the whole target passageway. We proposed and evaluated the availability of several our deployment method in field operation test in the past [24]-[26].

To keep the throughput of 1.0 [Mbps] in End-to-End communication, two communication qualities between adjacent SNs need to be maintained. One is the RSSI that values over $-86[\mathrm{dBm}]$ between two adjacent SNs (1-hop) communication are required. A wireless LAN module controlling the throughput speed constantly refers the RSSI for stability of the network connection. If the RSSI value is below -86 [dBm], the wireless LAN module controls the upper limit of throughput speed to 5.5 [Mbps]. Our proposed method also measures the RSSI value to predict the throughput speed control of the wireless LAN module. The other is the throughput that values over 6.0 [Mbps] between adjacent SNs (each 1-hop) are required for maintaining the throughput over 1.0 [Mbps] between End-to-End communications. Moreover, for the decision of SN deployment position, measurement of the throughput between the operator and the mobile robot as
End-to-End communication is required to evaluate the communication quality. Therefore, both the throughput and RSSI values must be measured to satisfy the required communication performance for mobile robot teleoperation, and the robot measures both the throughput and RSSI value between each SN accordingly. The robot moves continuously to the destination while maintaining the $1.0 \mathrm{Mbps}$ of throughput required for end-to-end communication and an RSSI value of $-86 \mathrm{dBm}$ between each SN(Fig. 2).

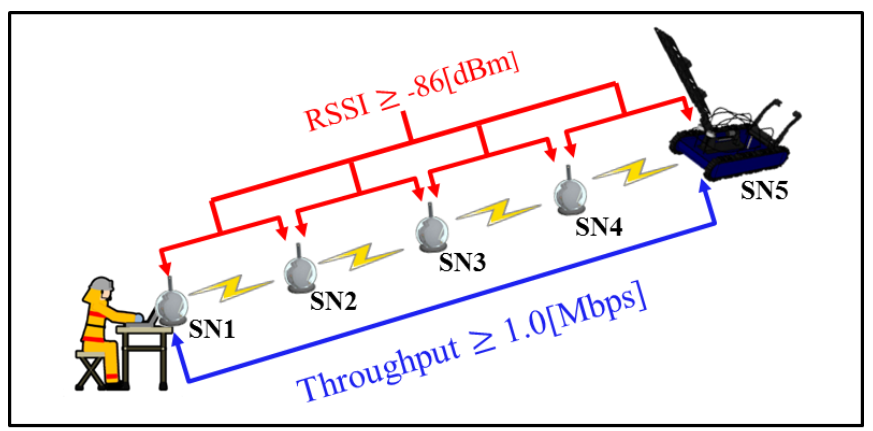

Fig. 2. SN Deployment method

\section{Routing Path Decision Method Based on Communication Connectivity Between Adjacent SNs}

As mentioned above, the proposed routing path decision method is necessary to prevent the communication disconnection and to maintain the throughput of over 1.0 [Mbps] between End-to-End communications.

In the network topology of the WSN constructed by the mobile robot, SNs are linearly connected and deployed. Then, the mobile robot moves on a straight line along deployed SN. At that time, the mobile robot communicates with a last deployed SN by 1-hop. Then, the distance between the mobile robot and this SN changes by moving the mobile robot. Generally, the throughput decays by degrading the RSSI and increasing the noise signal with the extension of distance between SNs. From this reason, it is necessary to decide the routing path between End-to-End communications flexibly against change of the distance between the SN and the mobile robot.

In order to maintain stability of the communication quality between each $\mathrm{SN}$ in the WSN constructed by our SN deployment method, we propose a decision method of a routing path between End-to-End communications based on relative positions of the mobile robot and SNs. When the positions of the mobile robot and a SN are the same, the mobile robot selects the $\mathrm{SN}$ of high connection priority, and then switches the routing path. A connection priority is different from the moving direction of the mobile robot. Each SN stores the routing path to the last deployed SN. Hence, using these routing path policies, the mobile robot can decide the routing path between End-to-End communications. Then, the throughput between End-to-End communications is increased or decreased depending on the number of hops by updating the routing path. Though, the proposed method maintains the throughput between End-to-End communications and prevents communication disconnection because the throughput and the RSSI value between SNs are stable. 
Figure 3 to 5 show the workflow of this routing path decision method to be applied to the mobile robot (SN5). In the workflow, $\mathrm{X}$ is parameter of deployed SN ID, and Y is parameter of the high primary connection SN ID (hpc-SN ID).

Also, we define the source direction is that the mobile robot moves back to the passage, and the destination direction is that the mobile robot moves forward to the passage.

Moreover, Table 1 and 2 show a pattern of routing path. The workflow is outlined below.

1) The mobile robot records the SN deployment position with referring to own odometry and assigns an ID to the $S N$ deployed one by one $(X=1,2,3,4)$.

2) The mobile robot moves along the constructed WSN. Then the mobile robot measures movement distance by referring to the odometry.

3) The mobile robot compares self-position and the $S N$ deployment position. If their positions match, the mobile robot refers to SN ID (choice SN ID, X). Otherwise, the workflow backs to the process (2).

4) The mobile robot identifies the forward direction and in case the mobile robot is moving the source direction (point of SNI: O [m]), the workflow progresses to the process (5). Otherwise (the destination direction: the mobile robot is facing the SN2, SN3, or SN4), the workflow progresses to the process (6).

5) For the movement to the source $S N$ (SN1) direction, the mobile robot selects the $S N(I D=X-1)$ which has high connection priority, and multicast the packet to run the command changing routing path. Then, the $S N$ which received the packet updates the routing table and connects to the mobile robot (Fig. 4).

6) For the movement to the destination $S N(S N 4)$ direction, the mobile robot selects the $S N$ that $S N(I D=X)$ which has high connection priority, and multicast the packet to run the command changing routing path. Then, the $S N$ which received the packet updates the routing table and connects to the mobile robot (Fig. 5).

7) The mobile robot send the packet to the SNI which is the source SN for the operator, and then each SN transfers received packet to the SN1 while updating the routing table. By repeating this, the mobile robot decides the routing path between End-to-End communications.

TABLE I. ROUTING PATH MOVEMENT To SOURCE DiRECTION

\begin{tabular}{|c|c|}
\hline Mobile Robot Position & Routing Path \\
\hline SN2 & SN1-SN5 \\
\hline SN3 & SN1-SN2-SN5 \\
\hline SN4 & SN1-SN2-SN3-SN5 \\
\hline
\end{tabular}

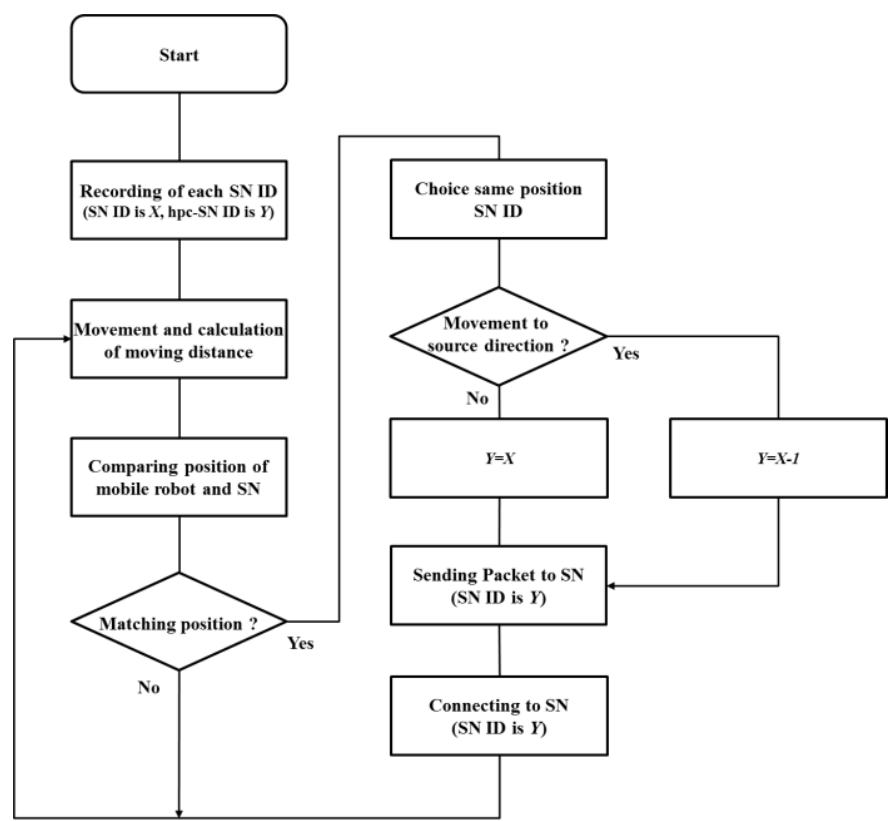

Fig. 3. Workflow of our proposed routing path decision method

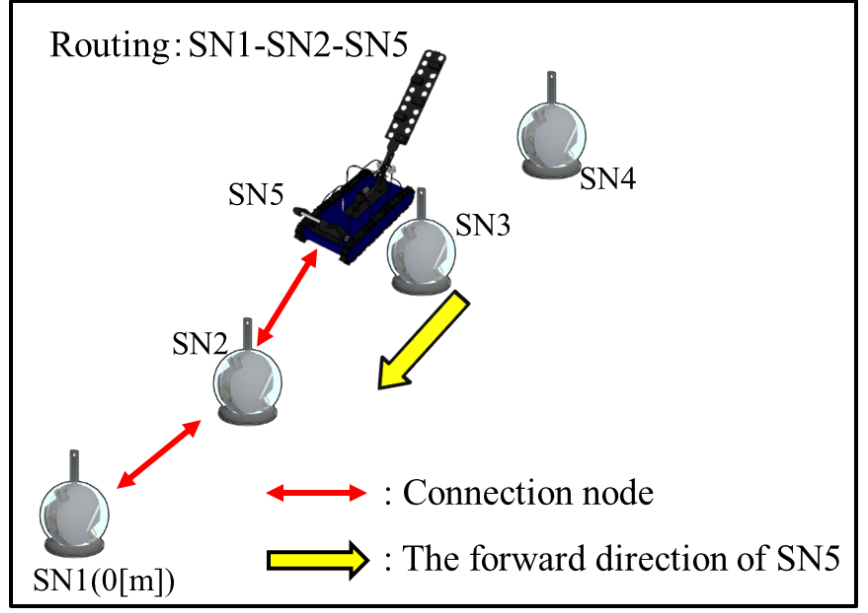

Fig. 4. Decision of routing path at source direction (e.g. mobile robot reaches point of SN3)

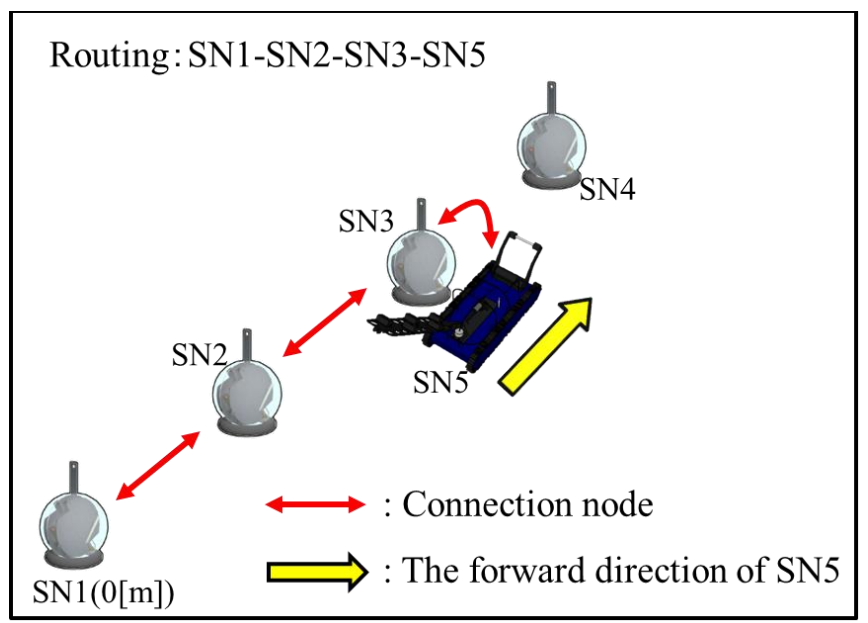

Fig. 5. Decision of routing path at destination direction (e.g. mobile robot reaches point of $\mathrm{SN} 3$ ) 
TABLE II. ROUTING PATH MOVEMENT TO DESTINATION DIRECTION

\begin{tabular}{|c|c|}
\hline Mobile Robot Position & Routing Path \\
\hline SN2 & SN1-SN2-SN5 \\
\hline SN3 & SN1-SN2-SN3-SN5 \\
\hline SN4 & SN1-SN2-SN3-SN4-SN5 \\
\hline
\end{tabular}

\section{Performance Evaluation of Proposed Routing METHOD}

\section{A. Experimental Conditions}

This section describes experimental conditions for performance evaluation of proposed routing method. Pseudo failure SN disconnects WSN between the operator and the mobile robot, and then proposed routing method reconstructs the network in this experiment. The experiment verifies maintaining connectivity by measuring throughput between End-to-End communications. Stability of the throughput indicates success of change of the routing pass, and then WSN reconstruction prevents the disconnection of teleoperation between the operator and the mobile robot. In this experiment, we performed a cooperative evaluation of a fixed linear routing method and the proposed routing method.

The experiment was performed in the passageway with a length of $300[\mathrm{~m}]$ or more in Tokyo Denki University, and the WSN was constructed by utilizing RWSN in this environment (Fig. 6). The SN configuring the WSN mounts the CPU board, memory device, CompactFlash disc, IEEE $802.11 \mathrm{~b} / \mathrm{g}$ wireless LAN module, and a battery. These devices are controlled by Linux OS (Debian) installed to the CPU board. By utilizing the "AODV-uu", Ad-Hoc networks can be constructed on the WSN. Table 3 shows the specification of the SN. The CPU board is "Armadillo-300" (@techno Inc.) in which Linux OS is implemented (Fig. 7 (a)). In RSSI value measurement, we used "wlanconfig" command included in the Linux wireless tools.

The throughput was measured by using "utest" provided by NTTPC Communications, Inc. The "utest" is a tool measuring throughput by transferring 10000 times of the packet of 1500 [byte] to communication partner. The throughput is calculated by received data per unit time. The crawler-type mobile robot, "S-90LWX" (TOPY INDUSTRIES, LIMITED) equipped with a SN deployment mechanism shown in Fig. 7 (b), is used as the mobile robot in this experiment.

The mobile robot constructed a WSN by utilizing our proposed SN deployment strategy prior to the evaluation. Each SN were deployed at SN1: 0 [m], SN2: 80 [m], SN3: 128 [m], SN4: 136 [m], and SN5: 196 [m]. After that, we measured 10 times of RSSI value and throughput between 1-hop connections in each $4[\mathrm{~m}]$ interval in two situations of the linear routing network and the proposed routing method while the mobile robot (SN5) moved from the point of 196 [m] to the point of $0[\mathrm{~m}]$. The operator controlled the mobile robot as SN5 connected to SN1 via the WSN. The WSN provided communication infrastructure to the operator for wireless teleoperation of the mobile robot in this experiment.

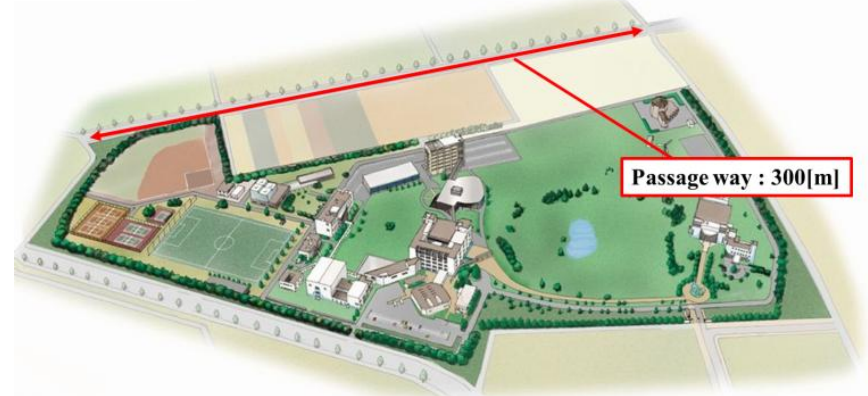

Fig. 6. Experimental environment

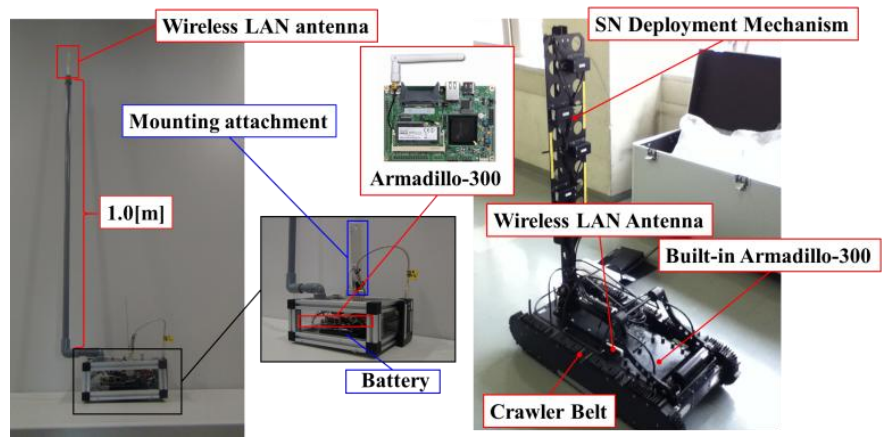

(a) Sensor Node

(b) S-90LWX

Fig. 7. Configuration devices on WSN

TABLE III. SPECIFICATION OF WIRELESS SENSOR NODE

\begin{tabular}{|c|c|}
\hline \multicolumn{2}{|c|}{ Sensor Node } \\
\hline Operating system & Linux Kernel 2.6 (Debian) \\
\hline CPU board & Armadillo-300 $($ ARM $200[\mathrm{MHz}])$ \\
\hline Weight & $1.5[\mathrm{~kg}]$ \\
\hline Height $\times$ Width $\times$ Length & $225[\mathrm{~mm}] \times 180[\mathrm{~mm}] \times 380[\mathrm{~mm}]$ \\
\hline Battery No. 1 & Output : $5[\mathrm{~V}], 1.8[\mathrm{~A}]$ \\
\hline Battery No. 2 & Output $: 12[\mathrm{~V}], 2.1[\mathrm{~A}]$ \\
\hline Operating time & $3[\mathrm{hour}]$ \\
\hline
\end{tabular}

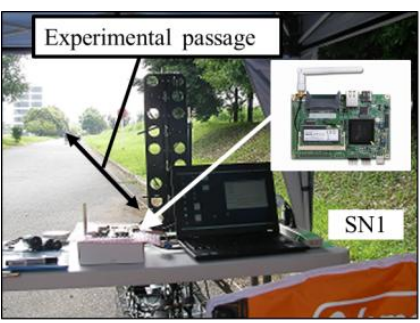

(a) Experimental place

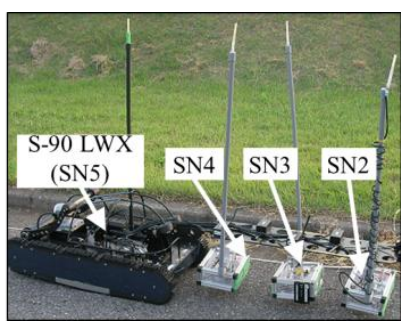

(b) S-90LWX with SN
Fig. 8. Overview of experimental environment

\section{B. Experimental Results}

Figure 9 and 10 show experimental results that are the mean value of RSSI and throughput in fixed linear routing network. Figure 11 and 12 show the values in proposed routing network. In fixed linear routing network, measurement intervals of RSSI and throughput were between SN4 and SN5. 
In the proposed routing network, SN4 connects SN5 between $196[\mathrm{~m}]$ and $136[\mathrm{~m}]$, SN3 connects SN5 between 132 [m] and $128[\mathrm{~m}]$, SN2 connects SN5 between $124[\mathrm{~m}]$ and 80 $[\mathrm{m}]$, and SN1 connects SN5 between $76[\mathrm{~m}]$ and $0[\mathrm{~m}]$ (Fig. 11 and 12). Thus measurement intervals of RSSI and throughput were above intervals in proposed routing network.

We confirmed that the mobile robot as SN5 was able to connect to WSN in all measurement intervals of the fixed linear routing network and the proposed routing network. Then the operator could control the mobile robot by connecting WSN in both routing network without communication failures such as network disconnection, multipath fading of radio wave, various environmental noise and so on.

\section{DISCUSSION}

In the case of using the fixed linear routing network in the performance evaluation, the throughput between End-to-End communications was lower than 1.0 [Mbps] at the point of 80 [m] (Fig. 9). It shows maintaining the throughput of 6.0 [Mbps] between SN4 and SN5 was difficult with the decrease of RSSI because the distance between SN4 and SN5 was expanded (Fig. 10). Also, the throughput between SN4 and SN5 was unstable from the point of over $80[\mathrm{~m}]$ where the upper limit value of the throughput was adjusted frequently. Then, the operability of the mobile robot might decrease.

Against that, the case of using our proposed routing method, the throughput between End-to-End communication increased from 1.4 [Mbps] to 7.0 [Mbps] because the number of hops between SN1 and SN5 decreased (Fig. 11). Therefore, we confirmed that the throughput of 1.0 [Mbps] between End-toEnd communications and 7.0 [Mbps] between the mobile robot and the SN connected by 1-hop were maintained at all SN deployed positions (Fig. 12). These results show the availability of our proposed method in this field test.

\section{CONCLUSION}

This paper proposed the WSN management method of changing routing path considering communication disconnection by the effect of multi path fading. The proposed method maintained communication conditions that throughput

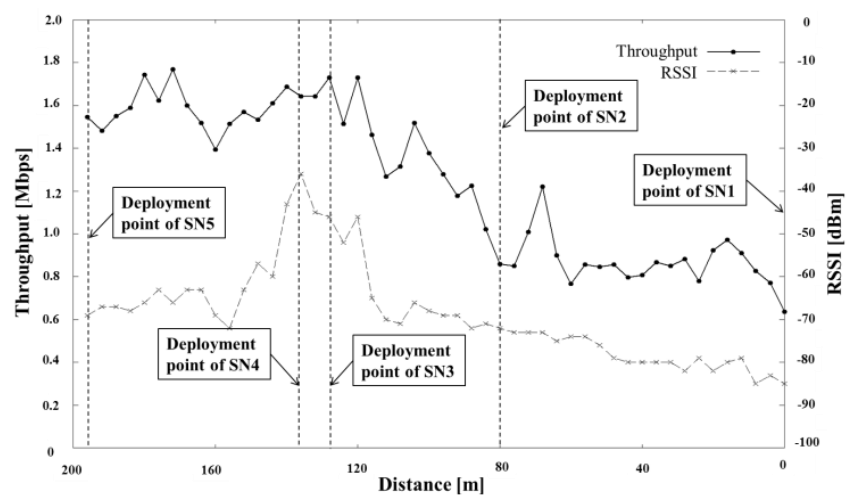

Fig. 9. Experimental result of RSSI and End-to-End throughput in fixed linear routing network

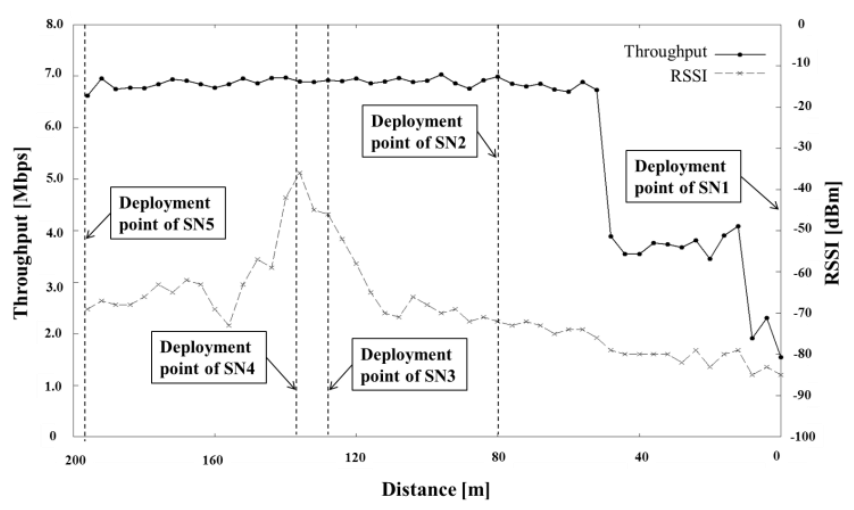

Fig. 10. Experimental result of RSSI and 1-hop throughput in fixed linear routing network

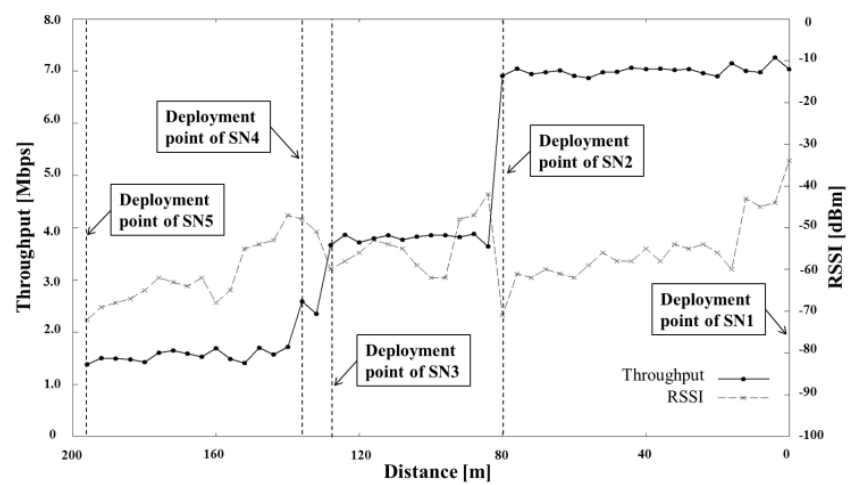

Fig. 11. Experimental result of RSSI and End-to-End throughput utilizing our proposed routing network

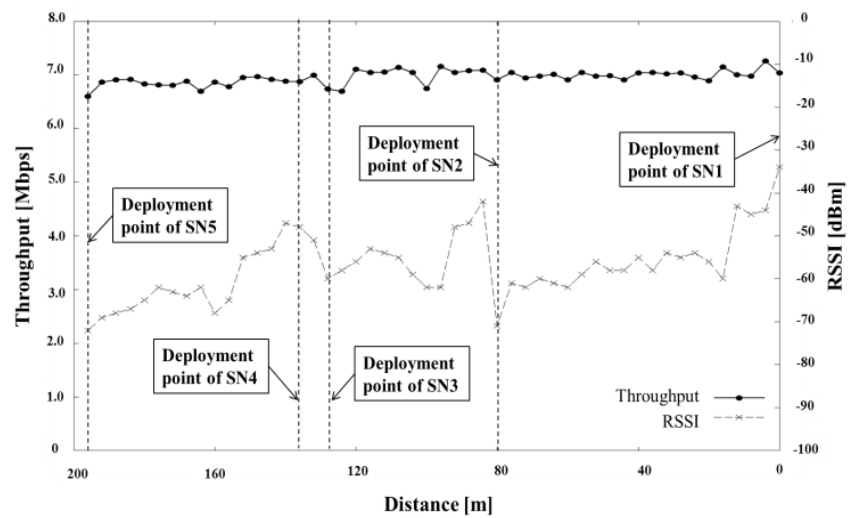

Fig. 12. Experimental result of RSSI and 1-hop throughput utilizing our proposed routing network

between End-to-End communications in the WSN enables smooth teleoperation of the mobile robot in a post-disaster underground space. Experimental results showed the effectiveness of the proposed method that enables to manage the WSN in the field test. The rapid implementation of actions to reduce secondary disasters in disaster areas requires the stable referral of disaster information. Therefore, this WSN managing method that maintains the throughput stable by utilizing the mobile robot is effective for gathering disaster area information in actual disaster scenarios. 
In future work, we should consider the communication disconnection on WSN due to $\mathrm{SN}$ failure. In the underground space, the SN may break down by the effect of secondary disasters. It means that information gathering by the RWSN becomes impossible. We will consider the improved SN redeployment method to repair the communication disconnection by integrating our proposed routing path decision methods.

\section{ACKNOWLEDGMENT}

This work was partially supported by the Research Institute for Science and Technology of Tokyo Denki University, Grant Number Za10-01 / Japan.

\section{REFERENCES}

[1] CHI Hao-yuan, LIU Xu, XU Xiao-dong, "A Framework for Earthquake Disaster Mitigation System," Proceedings of 2011 China located International Conference on Information Systems for Crisis Response and Management (ISCRAM), pp.490-495, 2011.

[2] Huang AN, "China's Emergency Management Mechanisms for Disaster Prevention and Mitigation," Proceedings of International Conference on E-Business and E-Government (ICEBEG), pp.2403-2407, 2010.

[3] Yoshiaki KANAEDA, Kazushige MAGATANI, "Development of the device to detect SPO2 in the Field,"31st Annual International Conference of the IEEE EMBS, pp.412-415, September 2009.

[4] O. Mizuno, A. Takashi, S. Yamamoto, and K. Asatani, "Sustainable operation technologies for the mitigation information network in urban area", In Humanitarian Technology Conference (R10-HTC) 2013 IEEE Region 10, pp. 255-260, 2013.

[5] A. P. Tang, and A. P. Zhao, "A Decision Supporting System for Earthquake Disaster Mitigation", Intelligent System Design and Engineering Application (ISDEA), 2012 Second International Conference on, IEEE, pp.748-751, 2012.

[6] M. Asif, T. Nitin, K. Ullah, and M.S. Sarfraz, "A Web-based Disaster Management-Mitigation Framework Using Information and Communication Technologies and Open Source Software", JU Journal of Information Technology (JIT), Vol.1, pp.15-18, 2012.

[7] Helge-Bjorn Kuntze, Christian W. Frey, Igor Tchouchenkov, Barbara Staehle, Erich Rome, Kai Pfeiffer, Andreas Wenzel and Jurgen Wollenstein, "SENEKA - Sensor Network with Mobile Robots for Disaster Management," Homeland Security (HST), pp.406-410, 2012.

[8] Abishek T K, Chithra K R and Maneesha V. Ramesh, "ADEN:Adaptive Energy Efficient Network of Flying Robots Monitoring over Disaster Hit Area," Proceedings of 8th IEEE International Conference on Distributed Computing in Sensor Systems (IEEE DCOSS), pp.306-310, 2012.

[9] E. Budianto, M.S. Alvissalim, A. Hafidh, A. Wibowo, W. Jatmiko, B. Hardian, P. Mursanto and A. Muis, "Telecommunication Networks Coverage Area Expansion in Disaster Area using Autonomous Mobile Robots : Hardware and Software Implementation," Proceedings of International Conference on Advanced Computer Science and Information Systems (ICACSIS), pp.113-118, 2011.

[10] Andrew Chiou, and Carol Wynn, "Urban Search and Rescue Robots in Test Arenas: Scaled Modeling of Disasters to Test Intelligent Robot Prototyping," Proceedings of International Conference on Autonomic and Trusted Computing (ATC), pp.200-205, 2009.

[11] R.C. Luo, O. Chen, "Mobile sensor node deployment and asynchronous power management for wireless sensor networks." Industrial Electronics, IEEE Transactions on, Vol.59, Issue.5, pp.2377-2385, 2012.

[12] Wing-Yue Geoffrey Louie, and Goldie Nejat, "A victim identification methodology for rescue robots operating in cluttered USAR environments," Advanced Robotics, vol. 27, issue. 5, pp. 373-384, 2013.

[13] Andrew Markham and Niki Trigoni, "Magneto-Inductive NEtworked Rescue System (MINERS):Taking Sensor Networks Underground," Proceedings of the 11th international conference on Information Processing in Sensor Networks (IPSN '12), pp. 317-328, 2012.

[14] Josh D. Freeman, Vinu Omanan, and Maneesha V. Ramesh, "Wireless Integrated Robots for Effective Search and Guidance of Rescue Teams," Proceedings of 8th International Conference on Wireless and Optical Communications Networks (WOCN 2011), pp. 1-5, 2011.

[15] A. Kumar, V. Sharma, and D. Prasad, "Distributed Deployment Scheme for Homogeneous Distribution of Randomly Deployed Mobile Sensor Nodes in Wireless Sensor Network." International Journal of Advanced Computer Science and Applications (IJACSA), The Science and Information organization, Vol.4, No.4, pp.139-146, 2013.

[16] Z. Wang, X. Zhao, and X. Qian, "Carrier-based sensor deployment by a mobile robot for wireless sensor networks", Control Automation Robotics \& Vision (ICARCV), 2012 12th International Conference on. IEEE, pp.1663-1668, 2012.

[17] C. Rizzo, D. Tardioli, D. Sicignano, L. Riazuelo, J. L. Villarroel, and L. Montano, "Signal-based deployment planning for robot teams in tunnellike fading environments", The International Journal of Robotics Research, Vol.32, No.12, pp.1381-1397, 2013.

[18] H. Sato, K. Kawabata and T. Suzuki, "Information Gathering by wireless camera node with Passive Pendulum Mechanism," International Conference on Control, Automation and Systems 2008 (ICCAS2008), pp.137-140, 2008.

[19] T. Yoshida, K. Nagatani, E. Koyanagi, Y. Hada, K. Ohno, S. Maeyama, H. Akiyama, K. Yoshida and S. Tadokoro, "Field Experiment on Multiple Mobile Robots Conducted in an Underground Mall," Field and Service Robotics Springer Tracts in Advanced Robotics, vol. 62, pp365$375,2010$.

[20] H. Jiang, J. Qian, and W. Peng, "Energy Efficient Sensor Placement for Tunnel Wireless Sensor Network in Underground Mine," Proceedings of 2nd International Conference on Power Electronics and Intelligent Transportation System (PElTS 2009), pp. 219-222, 2009.

[21] J. Xu, S. Duan and M. Li, "The Research of New Type Emergency Rescue Communication System in Mine Based on Wi-Fi Technology," Proceedings of IEEE 3rd International Conference on Communication Software and Networks (ICCSN), pp. 8-11, 2011.

[22] K. Nagatani, S. Kiribayashi, Y. Okada, K. Otake, K. Yoshida, S. Tadokoro, T. Nishimura, T. Yoshida, E. Koyanagi, M. Fukushima and S. Kawatsuma, "Emergency Response to the Nuclear Accident at the Fukushima Daiichi Nuclear Power Plants using Mobile Rescue Robots," Journal of Field Robotics, vol. 30, no. 1, pp. 44-63, 2013.

[23] J. Yamashita, K. Sawai, Y. Kimitsuka, T. Suzuki,Y. Tobe, "The design of direct deployment method of sensor nodes by utilizing a rescue robots in disaster areas," SICE Annual Conference 2008, pp183, 2B3-4, 2008.

[24] Tsuyoshi Suzuki, Kei Sawai, Hitoshi Kono and Shigeaki Tanabe, "Sensor Network Deployment by Dropping and Throwing Sensor Node to Gather Information Underground Spaces in a Post-Disaster Environment," Descrete Event Robot, iConcept PRESS, in Press. 2012.

[25] K. Sawai, H. Kono, S. Tanabe, K. Kawabata, T. Suzuki, "Design and Development of Impact Resistance Sensor Node for Launch Deployment into Closed Area," In international journal of sensing for industry(Sensor Review), Emerald Group Publishing Ltd., Vol. 32, pp. $318-326,2012$.

[26] S. Tanabe, K. Sawai and T. Suzuki, "Sensor Node Deployment Strategy for Maintaining Wireless Sensor Network Communication Connectivity," International Journal of Advanced Computer Science and Applications (IJACSA), The Science and Information organization, Vol.2, No. 12, pp.140 - 146, 2011. 